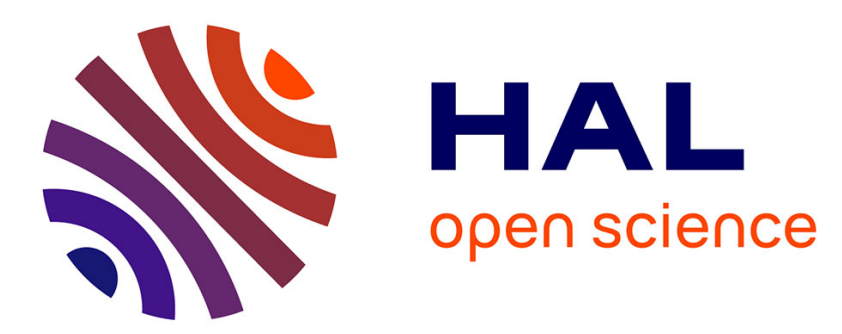

\title{
Optimization of Piezoelectric Sensors Location and Number Using a Genetic Algorithm
}

\author{
Isabelle Bruant, L. Gallimard, Shahram Nikoukar
}

\section{To cite this version:}

Isabelle Bruant, L. Gallimard, Shahram Nikoukar. Optimization of Piezoelectric Sensors Location and Number Using a Genetic Algorithm. Mechanics of Advanced Materials and Structures, 2011, 18 (7), pp.469 - 475. 10.1080/15376494.2011.604600 . hal-01689948

\section{HAL Id: hal-01689948 \\ https://hal.parisnanterre.fr/hal-01689948}

Submitted on 22 Jan 2018

HAL is a multi-disciplinary open access archive for the deposit and dissemination of scientific research documents, whether they are published or not. The documents may come from teaching and research institutions in France or abroad, or from public or private research centers.
L'archive ouverte pluridisciplinaire HAL, est destinée au dépôt et à la diffusion de documents scientifiques de niveau recherche, publiés ou non, émanant des établissements d'enseignement et de recherche français ou étrangers, des laboratoires publics ou privés. 


\title{
Optimization of Piezoelectric Sensors Location and Number Using a Genetic Algorithm
}

\author{
I. Bruant, L. Gallimard, and Sh. Nikoukar \\ Laboratoire LEME, EA 4416, Université Paris Ouest, Ville d'Avray, France
}

In this article, the optimal location and number of piezoelectric sensors is formulated for active vibration control. A modified criterion is used to ensure good observability of the system by considering the minimum number of needed piezoelectric elements. A genetic algorithm is proposed to solve this bi-objective optimization problem. Simulations are presented for a thin plate.

Keywords vibrations, piezoelectric sensors, optimization of sensors location and number, genetic algorithm

\section{INTRODUCTION}

In recent years, a great number of research results has been produced in active vibration control of flexible structures using piezoelectric actuators and sensors. It is obvious that misplaced sensors and actuators lead to problems, such as the lack of observability or controllability, which decreases strongly the performance of the control system. Many papers dealing with the optimization of actuators and sensors location can be found in the literature. An exhaustive review until 2001 is presented in Frecker [1].

Several cost functions are used for optimization of sensors locations. Hac and Liu [2], Jha and Inman [3], Bruant and Proslier [4] propose to maximize an observability criterion using the gramian matrice. In Gawronski [5], Qiu et al. [6] and Halim and Reza Moheimani [7], an optimal placement method using $H 2$ norm is presented. Hiramoto et al. [8] and Güney and Eskinat [9] suggest the simultaneous design of a computationally simple $H \infty$ controller and optimization of the location of sensors and actuators. In most of these papers, it is assumed that the number of sensors is a priori determined and very few papers deal with the choice of the number of piezo-electric devices.

In this article, we consider optimal design of the number and position of sensors. The modified optimization criterion developed in Bruant et al. [10] is used to optimize the location.

Received 15 September 2009; accepted 1 July 2010.

Address correspondence to I. Bruant, Laboratoire LEME, EA 4416, Université Paris Ouest, 50 rue de Sèvres, 92410, Ville d'Avray, France. E-mail: isabelle.bruant@u-paris10.fr

It ensures good observability of the structures. An observability index proposed in Bruant and Proslier [4] is introduced in order to minimize the sensors numbers. The optimal design problem is reduced to bi-objective non-linear minimization problems.

For such problems, the traditional algorithms (conjugate gradient, Newton-Raphson, etc.) are not applicable or tend to be trapped in local optima. In order to overcome these limitations, a stochastic optimization method [11], based on a genetic algorithm, was proposed in several papers, for example in [3,12-15]. One of the main advantages of these algorithms is that they do not have much mathematical requirements (i.e., gradient information) about the optimization problem (only evaluations of the objective function are needed). The genetic algorithms are conveniently presented using the metaphor of natural evolution: a randomly initialized population of individuals (i.e., a set of points of the search space) evolves following the principle of the survival of the fittest. New individuals are generated using simulated genetic operations such as mutation and crossover. The probability of survival of the new generated solutions depends on their fitness (how well they perform with respect to the optimization problem): the best are kept with a high probability and the worst are rapidly eliminated. We propose here an evolution of the Genetic Algorithm developed in Bruant et al. [10] that takes into account the number of piezoelectric sensors in a simple way.

In section 2 of this work, we point out the active vibration control equations and the classical formulation based on state space form. In section 3, the optimization criterion used for sensors locations is presented, the observability index is introduced and the bi-objective minimization problem is formulated. Then, in section 4, the GA method is briefly explained and a GA, optimizing the number as well as the location of the piezoelectric sensors is proposed. Finally in section 5, results are shown for a rectangular plate.

\section{EQUATIONS OF ACTIVE VIBRATION CONTROL}

Consider a flexible structure including $N_{a}$ piezoelectric actuators and $N_{s}$ piezoelectric sensors. According to the analytical model or finite element analysis, the equations of motion and the sensor's output equations of the system in modal coordinates 
can be written as:

$$
\begin{array}{rlrl}
\ddot{\alpha}_{i}+2 \zeta_{i} \omega_{i} \dot{\alpha}_{i}+\omega_{i}^{2} \alpha_{i} & =\sum_{l=1}^{N_{a}} b_{i l} \Phi_{l} & i & =1 \ldots N \\
y_{j} & =\sum_{l=1}^{N} c_{j l} \alpha_{l} & j & =1 \ldots, N_{s}
\end{array}
$$

where the $N$ first eigenmodes are considered. $\alpha_{i}, \dot{\alpha}_{i}$, and $\ddot{\alpha}_{i}$ represent modal displacement, velocity, and acceleration, $\omega_{i}$ and $\zeta_{i}$ are the natural frequency and damping ratio of the $i$-th mode, and $b_{i l} \Phi_{l}$ is the $i$-th modal component of the control force due to the electric potential $\Phi_{l}$ applied to the $l$-th actuator. $y_{j}$ is the quantity measured from the $j$-th sensor. $c_{j l}$ is the sensing constant of the $j$-th sensor due to the motion of the $l$-th mode. $b_{i l}, c_{j l}$, depend respectively of the $l$-th actuator location and $j$-th sensor location.

These equations can be written in a usual state-space form, using the state vector $\{x\}($ size $(2 N))$ :

$$
\begin{aligned}
\{x\} & =\left\{\omega_{i} \alpha_{i} \quad \dot{\alpha}_{i}\right\}^{T} \\
\frac{d}{d t}\{x\} & =[A]\{x\}+[B]\{\Phi\} \\
\{y\} & =[C]\{x\}
\end{aligned}
$$

where $[A]_{(2 N, 2 N)},[B]_{\left(2 N, N_{a}\right)}$, and $[C]_{\left(N_{s}, 2 N\right)}$ are the state, control, and output matrices given by:

$$
\begin{aligned}
{[A] } & =\left[\begin{array}{cc}
{[0]} & {\left[\omega_{i}\right]} \\
-\left[\omega_{i}\right]-\left[2 \zeta_{i} \omega_{i}\right]
\end{array}\right] \\
{[B]^{T} } & =\left[[0]\left[b_{i l}\right]\right] \\
{[C] } & =\left[\left[c_{j l}\right][0]\right]
\end{aligned}
$$

where $\{\Phi\}$ is the electric potential vector applied to the piezoelectric actuators. From Eq. (4), several automatic tools can be used to control the vibrations actively [16].

In order to control the motion of the $N$ first eigenmodes of the structure, the state of these modes must be well known: they must be well observed. In order to ensure good observability of each eigenmode, the best case should be:

$$
c_{j l}>0 \quad \forall j \in 1, \ldots, N_{s}, \quad \forall l \in 1 \ldots, N
$$

Hence, before setting up the regulator and observer system, the active elements' locations and numbers have to be determined. The following sections deal with the optimization of the sensors set.

\section{OPTIMIZATION OF THE SENSORS LOCATION AND NUMBER}

\subsection{The Optimization Criterion for Sensors Location}

In this work, the modified optimization criterion developed in Bruant and Proslier [4] is used. It deals with observability of each mode considering them with homogeneity and not globally as it is usually done: here, each mode is taken into account with the same magnitude. In this subsection, the number of sensors is assumed to be established.

The sensors are used to inform the active control system about the strain of the structure for desired modes. Their locations should be defined in order to maximize their output given by $\{y\}$. Consequently, most of the papers consider the maximization of the system output:

$$
\int_{0}^{\infty}\{y\}^{T}\{y\} d t .
$$

When the system is released from the initial state $\{x(0)\}=$ $\left\{x_{0}\right\}$, as when it is subjected to a persistent disturbance, Hac and Liu [2] have shown that maximizing the system output yields maximizing the gramian observability matrix defined by:

$$
\left[W_{o}\right]=\int_{0}^{\infty} e^{[A]^{T} t}[C]^{T}[C] e^{[A] t} d t
$$

where $\left[W_{o}\right]$ tends to a diagonal form:

$$
\begin{gathered}
\left(W_{o}\right)_{i i}=\left(W_{o}\right)_{i+N, i+N}=\sum_{j=1}^{N_{s}} \frac{c_{j i}^{2}}{4 \zeta_{i} \omega_{i}}=\frac{1}{4 \zeta_{i} \omega_{i}} \sum_{j=1}^{N_{s}} c_{j i}^{2} \\
i=1, \ldots, N
\end{gathered}
$$

In fact, each diagonal term $\left(W_{o}\right)_{i i}$ corresponds to the maximization of the output energy $J_{i}$ for the $i$-th mode obtained when the state equation is reduced to the $i$-th mode:

$$
\begin{aligned}
{\left[\begin{array}{c}
\omega_{i} \dot{\alpha}_{i} \\
\ddot{\alpha}_{i}
\end{array}\right] } & =\left[\begin{array}{cc}
0 & \omega_{i} \\
-\omega_{i} & -2 \zeta_{i} \omega_{i}
\end{array}\right]\left[\begin{array}{c}
\omega_{i} \alpha_{i} \\
\dot{\alpha}_{i}
\end{array}\right]+\left[\begin{array}{c}
{[0]} \\
{\left[b_{i l}\right]}
\end{array}\right]\{\Phi\} \\
\{y\} & =\left[C_{i}\right]\left[\begin{array}{c}
\omega_{i} \alpha_{i} \\
\dot{\alpha}_{i}
\end{array}\right] \quad J_{i}=\int_{0}^{\infty}\{y\}^{T}\{y\} d t .
\end{aligned}
$$

Consequently, if the $i$-th eigenvalue of $\left[W_{o}\right]$ is small, it means that the $i$-th mode will not be observed well.

Moreover, as the components of $\left[W_{o}\right]$ do not have the same order of magnitude, to find the sensors locations $x_{s}=\left(S_{1}, \ldots, S_{N_{s}}\right)$, maximizing

$$
\min _{i=1, \ldots, N}\left(W_{o}\left(x_{s}\right)\right)_{i i}
$$

can induce the study of particular modes instead of each of them. The obtained locations will not be optimal. 
Hence, to establish homogeneity between each term $\left(W_{o}\right)_{i i}$, we have suggested in Bruant and Proslier [4] to divide each of them by its maximal value obtained when the i-th mode is the specific mode to be measured. Then, the optimization problem considered here is to find the sensors locations $x_{s}=\left(S_{1}, \ldots, S_{N_{s}}\right)$ which maximize

$$
J_{S}\left(x_{s}\right)=\min _{i=1, N} \frac{\left(W_{o}\left(x_{s}\right)\right)_{i i}}{\max _{x_{s}}\left(W_{o}\left(x_{s}\right)\right)_{i i}}=\min _{i=1, N} \frac{\sum_{j=1}^{N_{s}} c_{j i}^{2}}{\max _{x_{s}} \sum_{j=1}^{N_{s}} c_{j i}^{2}}
$$

with $\forall i=1, \ldots, N, \quad 0 \leq \frac{\left(W_{o}\left(x_{s}\right)\right)_{i i}}{\max _{x_{s}}\left(W_{o}\left(x_{s}\right)\right)_{i i}} \leq 1$.

In this approach, all modes are normalized. $\max \left(W_{o}\left(x_{s}\right)\right)_{i i}$ represents the maximal output energy, which could be measured by the sensors for the i-th mode.

In the case where $N_{i}$ eigenmodes must be more observable than $N_{o}$ other ones (because for example, they are the most excited modes $)$, a weighting constant $(0<\gamma<1)$ can be used in $J_{S}$ :

$$
J_{S}\left(x_{s}\right)=\min _{i=1, N} \frac{\sum_{j=1}^{N_{i}} c_{j i}^{2}}{\max _{x_{s}} \sum_{j=1}^{N_{i}} c_{j i}^{2}}+\gamma \min _{i=1, N} \frac{\sum_{j=1}^{N_{o}} c_{j i}^{2}}{\max _{x_{s}} \sum_{j=1}^{N_{o}} c_{j i}^{2}} .
$$

In the same way, residual modes can be considered in the criterion (see, for example, [10]).

\subsection{Optimization of the Sensors Number}

Usually, the user fixes the number of patches. In this subsection, we suggest a simple tool that allows to automatically determine the number of sensors. Following the approach proposed in Bruant and Proslier [4], we introduce an observability index on the $i$-th mode:

$$
\mathcal{O}_{i}\left(x_{s}\right)=\frac{W_{o}\left(x_{s}\right)_{i i}}{\max _{S} W_{o}(S)_{i i}}
$$

with $\mathcal{O}_{i}\left(x_{s}\right) \geq 1$ if the $i$-th mode is better observed by the $N_{s}$ sensors than by one sensor $S$ ideally located for this eigenmode. To correctly observe each mode, we look for a sensor configuration $x_{s}$, such that

$$
\forall i \in 1, \ldots, N_{s} \quad \mathcal{O}_{i}\left(x_{s}\right) \geq \eta
$$

with $\eta \in] 0,1]$. The coefficient $\eta$ is set by the user to choose the minimum degree of observability allowed for each mode.

\subsection{The Optimization Problem}

The optimization problem consists in finding the number $N_{s}$ and the location of the sensors $x_{s}$, which minimize the number
TABLE 1

Geometrical characteristics of the plate and the piezoelectric patch

\begin{tabular}{lcc}
\hline & Plate & Piezoelectric \\
\hline Length $(\mathrm{m})$ & 0.3 & 0.02 \\
Width $(\mathrm{m})$ & 0.2 & 0.0133 \\
Thickness $(\mathrm{m})$ & 0.002 & 0.0001 \\
\hline
\end{tabular}

of sensors and maximize the function $J_{S}$ (Eq. (15)) subject to the constraint $\mathcal{O}_{i}\left(x_{s}\right) \geq \eta$. A priori, this problem is a bi-objective optimization problem and its solution is a set of Pareto points. However, it seems reasonable to choose a solution in the Pareto front that minimizes the number of sensors. This choice leads to a much simpler problem to solve than the complete bi-objective problem.

The new optimization problem is:

1. Find the minimum sensors number $N_{s}$ such that

$$
\max _{x_{s}} \min _{i} \mathcal{O}_{i}\left(x_{s}\right) \geq \eta
$$

2. For the number of sensor's $N_{s}$ found in step 1, find the sensor's locations $x_{s}=\left\{S_{1}, S_{2}, \ldots, S_{N_{s}}\right\} \in \mathcal{C}$, which maximize

$$
J_{S}\left(x_{s}\right)=\min _{i=1, N} \frac{\left(W_{o}\left(x_{s}\right)\right)_{i i}}{\max _{x_{s}}\left(W_{o}\left(x_{s}\right)\right)_{i i}},
$$

with the following inequality constraints:

$$
\mathcal{C}=\left\{x_{s} \text { such that } \forall i \in 1, \ldots, N_{s} \quad \mathcal{O}_{i}\left(x_{s}\right) \geq \eta\right\}
$$

The verification of condition (16) ensures that $\mathcal{C}$ is not an empty set.

\section{OPTIMIZATION IMPLEMENTATION USING GENETIC ALGORITHMS}

To solve the optimization problem defined by Eqs. (16) and (17), we use a modified version of the Genetic Algorithm proposed in Bruant et al. [10] for a fixed number of sensors. GAs are a family of optimization algorithms that evolve in an analogous manner as the Darwinian principle of natural selection. Several authors have used them for optimal location of sensors (see, for example, $[3,12-15])$.

\subsection{Genetic Algorithms}

Here, the use of a GA for the sensor's location optimization is recalled. The optimization variables are the coordinate of each sensor's center $\left(x^{s}, y^{s}\right)$.

GAs are derived from the mechanics of natural selection and genetics. They are an effective numerical method to 
TABLE 2

Mechanical characteristics of piezoelectric patch Zirconate P1 88

\begin{tabular}{lc}
\hline$\rho\left(\mathrm{kg} / \mathrm{m}^{3}\right)$ & 7650 \\
$C_{11}=C_{22}(\mathrm{GPa})$ & 132.2 \\
$C_{12}=C_{21}(\mathrm{GPa})$ & 82.3 \\
$C_{13}=C_{23}=C_{31}=C_{32}(\mathrm{GPa})$ & 83.7 \\
$C_{33}(\mathrm{GPa})$ & 120 \\
$C_{44}=C_{55}(\mathrm{GPa})$ & 29.5 \\
$C_{66}(\mathrm{GPa})$ & 25 \\
$\epsilon_{0}(\mathrm{~F} / \mathrm{m})$ & $8.85 e^{-12}$ \\
$\epsilon_{11}=\epsilon_{22}$ & $1440 \epsilon_{0}$ \\
$\epsilon_{33}$ & $837 \epsilon_{0}$ \\
$e_{31}=e_{32}\left(\mathrm{Cm}^{-2}\right)$ & -4.3 \\
$e_{33}\left(\mathrm{Cm}^{-2}\right)$ & 16.7 \\
$e_{15}\left(\mathrm{Cm}^{-2}\right)$ & 11.8 \\
Maximal value of $\left(q_{\phi}\right)(V)$ & 150 \\
\hline
\end{tabular}

find an optimal (or sub-optimal) solution to a complicated multi-parameter optimization problem, without calculating the derivatives of the function to be optimized. Basically, GA finds the optimal solution through iterating the GA operations on a population, which consists of a number $N_{i}$ of candidate solutions to the optimization problem.

Vocabulary of natural genetics is used in GA:

- The considered population contains $N_{i}$ individuals.

- One individual represents a candidate solution of the optimization problem. In our work, it consists of the set of $N_{s}$ sensors. Each individual is defined by $N_{s}$ chromosomes.

- A chromosome here is the location of one sensor.

- Each chromosome is defined with a sequence of two genes: the coordinates of the sensor's center.

Several representations for genes can be used (binary or realencoded). Here, we use the real-encoded GA [13].

The GA method starts with a randomly generated population. At each iteration, a new population is created by repeating the following steps:

- Selection: Select two parent individuals from the population according to their fitness value: the better the fitness, the bigger the chance to be selected. Here, the fitness is the criteria $J_{S}$.

- Crossover: Using arithmetical crossover [13], the two selected parents give two children.
TABLE 3

Mechanical characteristics of the elastic plate

\begin{tabular}{lc}
\hline$\rho\left(\mathrm{kg} \mathrm{m}^{-3}\right)$ & 7870 \\
$\mathrm{E}(\mathrm{GPa})$ & 207 \\
$\nu$ & 0.292 \\
$\zeta_{i}$ & 0.0001 \\
\hline
\end{tabular}

- Mutation: With a mutation probability, some genes of the children are changed randomly.

- The new offspring are placed in the new population.

The crossover makes the GA process move in a desirable direction, and the mutation helps to prevent the process from getting trapped in a local optimal solution.

A conservation step (or elitism) is added and applied to the new generation: it consists of keeping the best parent (i.e., the greatest fitness value) in this new generation.

In practice, a convergence criterion must be specified. In this article, the GA is stopped if the best individual in the population does not change in continuous $N_{t o l}$ operations.

Of course, the results obtained from the GA process might be a suboptimal solution. To get a result with higher confidence, one has to run the GA process several times, each with a randomly generated initial location [14].

\subsection{A Semi-Genetic Algorithm}

The algorithm proposed in this article is a semi-genetic algorithm (SGA). In a first step, the observability index is used to estimate the minimum number of sensors to obtain:

$$
\mathcal{O}_{i}\left(x_{s}\right) \geq \eta \quad \text { for each } i \in\{1, \ldots, N\} .
$$

In a second step, the optimal solution for this number of sensors is computed. The flowchart of this algorithm is detailed in Figure 1. The proposed SGA performs several runs of the GA for a fixed number of sensors. The initial number of sensors $N_{s}$ is set to $N$ (the number of modes to be observed) and the GA is run with a number of sensors fixed to $N_{s}$. However, the algorithm stops as soon as condition (18) is obtained for one set of sensors. The number of sensors is then decreased by one. This procedure continues until we obtain convergence of the GA before condition (18) is verified. The optimal number of sensor's is set to $N_{o p t, s}=N_{s}+1$, and the GA is until convergence occurs.

TABLE 4

The eight first frequencies of the structure

\begin{tabular}{lcccccccc}
\hline Number of frequency & 1 & 2 & 3 & 4 & 5 & 6 & 7 & 8 \\
Value $(\mathrm{Hz})$ & 175.57 & 337.57 & 539.97 & 607.55 & 701.90 & 971.83 & 985.71 & 1146.7 \\
\hline
\end{tabular}




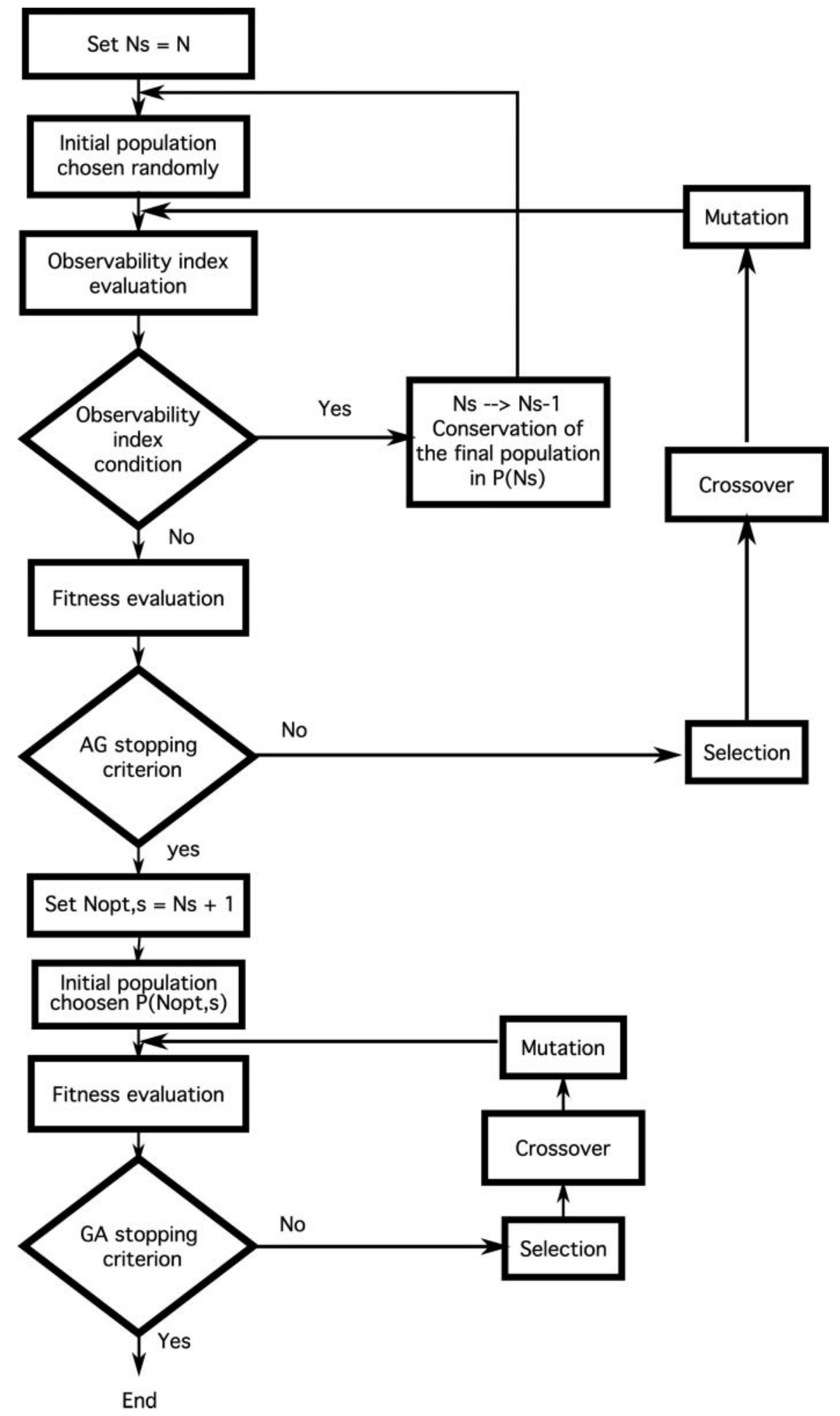

FIG. 1. Flowchart of the semi-genetic algorithm. 


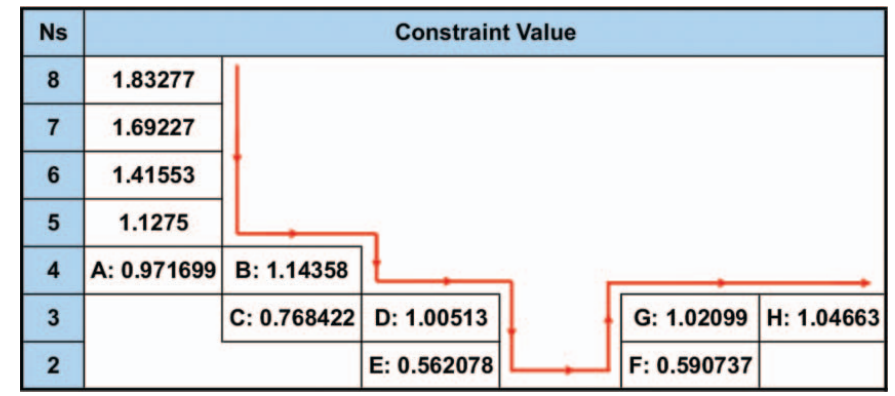

FIG. 2. Values of the observability index condition: $\min _{i=1, N} \mathcal{O}_{i}$ (Color figure available online).

\section{Remark}

In practice, we randomly choose the initial population in each step of the SGA, except in the last step where the population conserved at the end of the step $N_{o p t, s}$ is used to start the final GA search.

\section{APPLICATION}

In this section, the optimization process is applied in the case of a simply supported elastic plate equipped with piezoelectric sensors. In order to consider only pure bending motion, each sensor is made up of a pair of the same piezoelectric material attached symmetrically. They are assumed to be perfectly bonded to the surface of the plate. The geometrical and mechanical properties of the system are detailed in Tables $1-3$.

The plate is modeled using a new piezoelectric FE (Finite Element), which has been developed and validated in Polit and Bruant [17] and used for active vibration control in Bruant et al. [18]. The plate is discretized in $15 \times 15$ elements. The
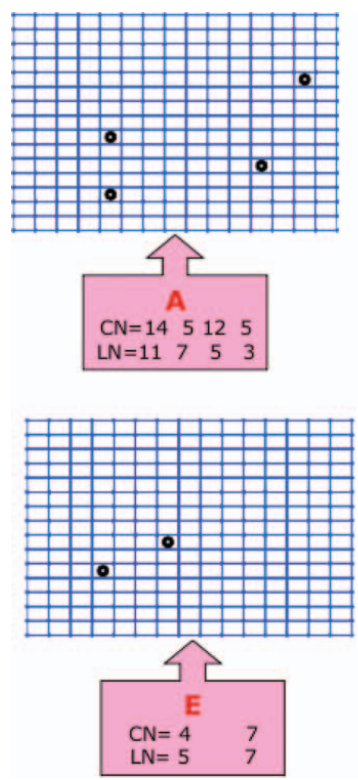
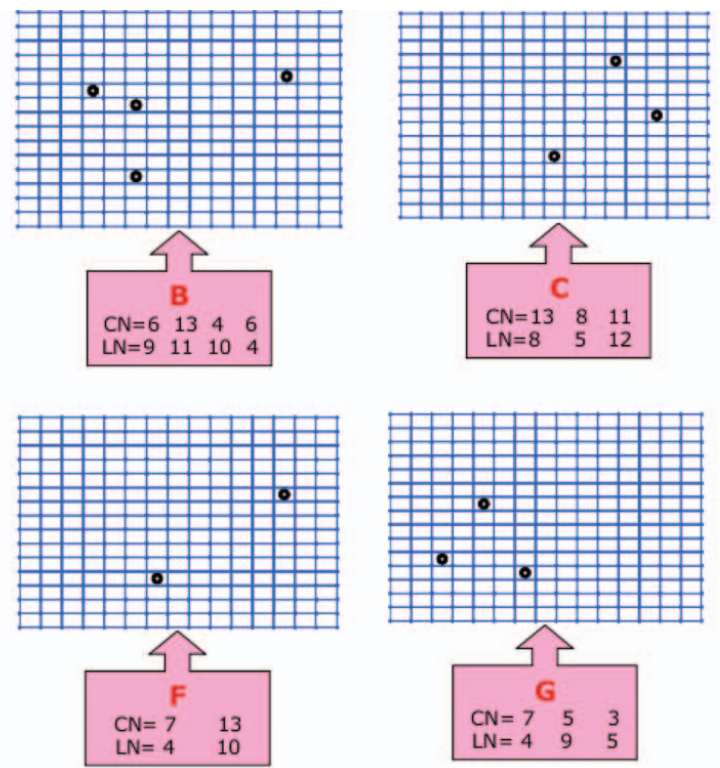

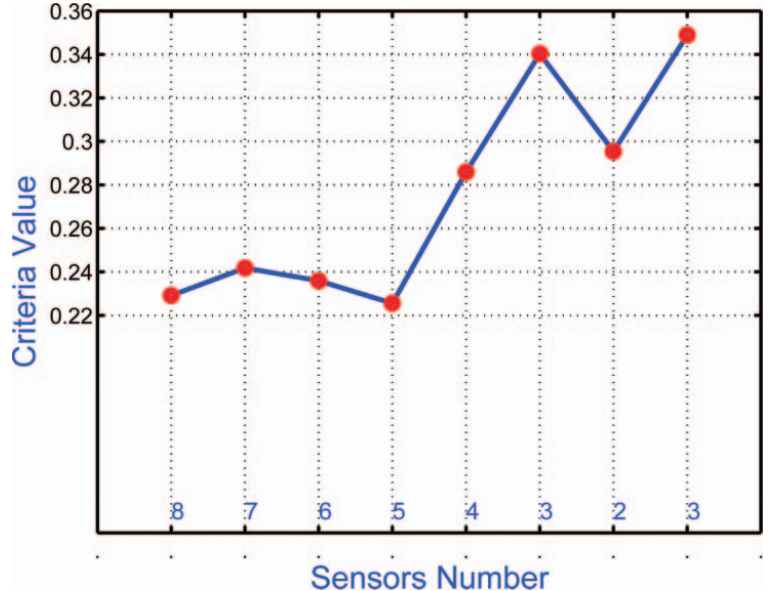

FIG. 4. Value of the criteria $J_{S}$ for each sensor's number (Color figure available online).

eigenfrequencies are given in Table 4. The parameters of the GA are as follows: the population size, the crossover probability, the mutation probability, and the number $N_{t o l}$ are as $16,100 \%$, $10 \%$ and 20 , respectively. The value of parameter $\eta$ is fixed to 1 .

The validation of the GA for optimization of the sensor's location and its convergence have been shown in Bruant et al. [10].

Here, the number and locations of piezoelectric sensors are optimized to observe well the 8 first eigenmodes. For this application, the SGA is run 20 times. The mean value, standard deviation, and best and worst values of $J_{S}$ are given Table 5 . In conclusion, the best value of $J_{S}$ equals to 0.349 and the value of the observability criteria condition equals to 1.046 with 3 sensors. It means that the observability of the 8 first
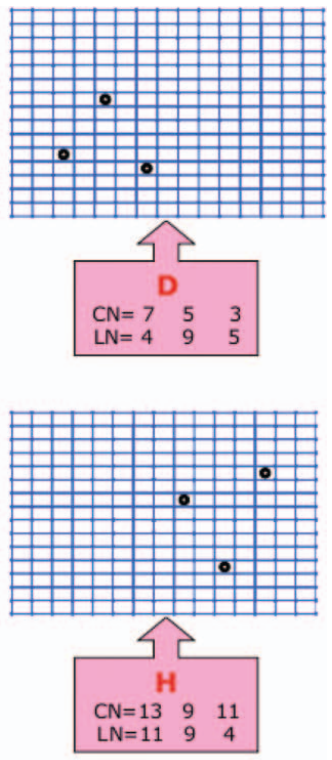

FIG. 3. Optimal location for each optimization step shown in Figure 2. CN: column number; LN: line number (Color figure available online). 
TABLE 5

Results about $J_{S}$ for 20 times running

\begin{tabular}{llcc}
\hline & Standard & & \\
Mean value & deviation value & Best value & Worst value \\
0.346 & $4.26 e^{-3}$ & 0.349 & 0.335 \\
\hline
\end{tabular}

eigenmodes, using 3 sensors, is good as in the case where each mode is observed by one sensor located optimally for itself. In the following paragraph, the results are detailed for one run.

According to the considered algorithm, the research process starts by running the GA with 8 sensors (the initial number of sensors equal to the number of eigenmodes). During the GA steps, as soon as the value of the observability index condition is greater or equal to $\eta=1$, the sensor's number is decreased by one. From $N_{S}=8$ to $N_{S}=3$, this condition value is verified (see Figure 2). For $N_{S}=2$, no optimal location is found ensuring the observability condition. Then, the number of sensors is fixed to $N_{\text {opt }, s}=3$, and the second part of the algorithm is applied to optimize the last configuration of 3 sensors. The optimal configurations at each step of the optimization process (from step $N_{S}=4$ ) are shown in Figure 3, the location of each sensor is defined by its column number $\mathrm{CN}$ and line number $\mathrm{LN}$. The maximal value of the optimization criteria $J_{S}$ is plotted in Figure 4 .

\section{CONCLUSIONS}

This work deals with the optimization of the sets of sensors. Usually, the number of sensors is fixed, but here it is considered as an optimization variable. A modified optimization criterion and an observability index condition are used to ensure good observability of all desired eigenmodes. A semi-genetic algorithm optimization is developed giving the optimal number and locations of sensors. Hence, in the studied application where the 8 first eigenmodes of a simply supported plate have to be observable, the number of piezoelectric sensors decreases from 8 to 3 . With only 3 sensors optimally located, each 8 first modes is well observed as with one sensor ideally located for him.

\section{REFERENCES}

1. M. Frecker, Recent advances in optimization of smart structures and actuators, J. Intell. Mat. Sys. Struct., vol. 14, pp. 207-215, 2003.

2. A. Hac and L. Liu, Sensor and actuator location in motion control of flexible structures, J. Sound Vibra., vol. 167, no. 2, pp. 239-261, 1993.

3. A.K. Jha and D.J. Inman, Optimal sizes and placements of piezoelectric actuators and sensors for an inflated torus, J. Intell. Mat. Syst. Struct., vol. 14, pp. 563-576, 2003.

4. I. Bruant and L. Proslier, Optimal location of actuators and sensors in active vibration control, J. Intell. Mat. Syst. Struct., vol. 16, pp. 197-206, 2005.

5. W. Gawronski, Simultaneous placement of actuators and sensors, J. Sound Vibra., vol. 228, no. 4, pp. 915-922, 1999

6. Z.-C. Qiu , X.-M. Zhang, H.-X. Wu, and H.-H. Zhang, Optimal placement and active vibration control for piezoelctric smart flexible cantilever plate, $\mathrm{J}$. Sound Vibra., vol. 301, pp. 521-543, 2007.

7. D. Halim and S.O. Reza Moheimani, An optimization approach to optimal placement of collocated piezoelectric actuators and sensors on a thin plate, Mechatronics, vol. 13, pp. 27-47, 2003.

8. K. Hiramoto, H. Doki, and G. Obinata, Optimal sensor/Actuator placement for active vibration control using explicit solution of algebraic Riccati equation, J. Sound Vibra., vol. 229, no. 5, pp. 1057-1075, 2000.

9. M. Güney and E. Eskinat, Optimal actuator and sensor placement in flexible structures using closed-loop criteria, J. Sound Vibra., vol. 312, pp. 210-233, 2007.

10. I. Bruant, L. Gallimard, and S. Nikoukar, Optimal piezoelectric actuator and sensor location for active vibration control, using genetic algorithm, J. Sound Vibra., 2010, online.

11. Z. Michalewicz, D. Dasgupta, R. Le Riche, and M. Schoenauer, Evolutionary algorithms for constrained engineering problems, Comput. Engineer., vol. 30, no. 4, pp. 851-870, 1996.

12. J.-H. Han and I. Lee, Optimal placement of piezoelectric sensors and actuators for vibration control of a composite plate using genetic algorithms, Smart Mat. Struct., vol. 8, pp. 257-267, 1999.

13. Y. Yang, Z. Jin, and C. Kiong So, Integrated optimal design of vibration control system for smart beams using genetic algorithms, J. Sound Vibra., vol. 282, pp. 1293-1307, 2005.

14. W. Liu, Z. Hou, and M.A. Demetriou, A computational scheme for the optimal sensor/actuator placement of flexible structures using spatial H2 measures, Mech. Syst. Signal Process., vol. 20, pp. 881-895, 2006.

15. K. Ramesh Kumar and S. Narayanan, Active vibration control of beams with optimal placement of piezoelectric sensors/actuator pairs, Smart Mat. Struct., vol. 17, no. 5, p. 055008, 2008.

16. A. Preumont, Vibration control of active structures, Kluwer Academic Publishers, Dordrecht, 1999.

17. O. Polit and I. Bruant, Electric potential approximations for an eight node plate finite element, Compos. Struct., vol. 84, pp. 1480-1493, 2006.

18. I. Bruant, F. Pablo, and O. Polit, Active Control of laminated plates using piezoelectric finite element, Mech. Adv. Mat. Struct., vol. 15, pp. 276-290, 2008. 